\title{
Variables ambientales durante blanqueamiento coralino en el Caribe colombiano
}

\author{
Deisy Romero-Rodríguez ${ }^{1, *}$, Gladys Bernal², Sven Zea ${ }^{3}$ \\ ${ }^{1}$ Instituto de Investigaciones Marinas y Costeras “José Benito Vives de Andréis”, INVEMAR, Santa Marta, Colombia \\ ${ }^{2}$ Departamento de Geociencias y Medio Ambiente, Universidad Nacional de Colombia, Medellín, Colombia \\ ${ }^{3}$ Centro de Estudios en Ciencias del Mar, CECIMAR, Universidad Nacional de Colombia, Sede Caribe, Santa Marta, Colombia
}

\begin{abstract}
Resumen
Aunque la temperatura superficial del mar se ha considerado como la variable ambiental con mayor incidencia en el blanqueamiento coralino, se ha encontrado que otras variables también intervienen en este fenómeno, modificando la susceptibilidad de los arrecifes y generando diferencias espaciales. En el Caribe colombiano se estudiaron las variables de temperatura superficial del mar, clorofila, turbidez, caudal de ríos, vientos y nubosidad durante los blanqueamientos coralinos en cuatro localidades. Mediante análisis multivariados y de valores extremos, se encontró que los blanqueamientos han ocurrido principalmente en el segundo semestre del año a nivel de cuenca, de costa continental y local. En los arrecifes costeros, la turbidez del agua por la entrada de agua dulce ha sido determinante en el blanqueamiento, mientras que en los oceánicos lo ha sido el aumento de temperatura. Así, fenómenos macroclimáticos como La Niña influyen en la ocurrencia de los blanqueamientos cerca de la costa. Los datos muestran que es necesario monitorizar de forma más continua el blanqueamiento y que las zonas más expuestas son las Islas del Rosario y San Bernardo.
\end{abstract}

Palabras claves: arrecifes coralinos, blanqueamiento, Caribe.

Environmental variables during coral bleaching in the Colombian Caribbean

\begin{abstract}
While sea surface temperature has been considered the environmental variable with the greatest impact on coral bleaching, other variables also modify the susceptibility of reefs and generate spatial differences of this phenomenon. In four locations in the Colombian Caribbean, sea surface temperature, chlorophyll, turbidity, flow of rivers, winds and clouds were studied during coral bleaching. Using a principal components and maximum values analysis, we found that bleaching occurred mainly in the second half of the year, at three spatial levels: basin, coastal zone and local. In the coastal reefs, water turbidity by freshwater input was determinant in bleaching, while in the open ocean, temperature was the determinant variable. Thus, macroclimatic phenomena like La Niña influence the occurrence of bleaching near the coast. The data backed the need to increase the frequency of bleaching monitoring and showed that the most exposed areas are El Rosario and San Bernardo islands.
\end{abstract}

Key words: Reefs, bleaching, Caribbean.

\section{Introducción}

El blanqueamiento coralino se produce cuando se trastorna la relación simbiótica entre los corales hermatípicos y las zooxantelas y estas son expulsadas, lo que deja expuesto el color blanco del esqueleto del coral (Douglas, 2003; Buddemeier, et al., 2004). Los efectos conocidos del blanqueamiento incluyen la pérdida de pigmentos fotosintéticos, el incremento en la tasa de respiración y la disminución de la calcificación (Brown, 1997). La temperatura superficial del mar (TSM) ha sido considerada como la principal causa del blanqueamiento (Glynn, 1993, Wilkinson \& Souter, 2008), debido a que los corales viven cerca de su límite de tolerancia térmica (Kleypas, et al., 2001). También se ha asociado el blanqueamiento con cambios en la salinidad, pues ésta modifica la función metabólica de los corales y sus algas simbiontes (Hoegh-Guldberg, 1999, True, 2012). Asimismo, el exceso de nutrientes puede producir un crecimiento acelerado de macroalgas, las cuales recubren los corales,

\section{*Correspondencia:}

Deisy Romero-Rodríguez, dalejandra86@gmail.com

Recibido: 22 de junio de 2014

Aceptado: 29 de octubre de 2014 
generando blanqueamiento (True, 2012; Wiedenmann, et al., 2013). Los eventos extremos, como los huracanes, también pueden estar indirectamente relacionados, ya que producen la fragmentación de la cobertura coralina (Van Woesik, et al., 1995; Gardner, et al., 2005). De otro lado, se ha sugerido que la nubosidad puede disminuir la alta radiación solar que reciben los corales (Mumby, et al., 2001) y la surgencia puede retardar el recalentamiento y permitir una rápida recuperación luego del blanqueamiento (Maina, et al., 2008, Bayraktarov, et al., 2013).

En 1997, durante El Niño, ocurrió un blanqueamiento masivo en el cual el $16 \%$ de los arrecifes del Caribe resultaron deteriorados (Wilkinson \& Souter, 2008). En 1998, se presentó blanqueamiento en la isla de San Andrés, en Santa Marta y en las islas del Rosario y, aunque la causa precisa no se determinó, este se atribuyó al incremento de la temperatura superficial del mar (TSM) (Rodríguez-Ramírez, et al., 2006; Wilkinson \& Souter, 2008). En el 2005, el blanqueamiento masivo en el Caribe también fue relacionado con el incremento de la TSM; en las islas del Rosario y de San Bernardo se reportó que en el 70-80 \% de la cobertura de coral hubo señales de blanqueamiento (Rodríguez-Ramírez, et al., 2008; Eakin, et al., 2010) y en el $8 \%$ hubo mortalidad (Gil-Agudelo, et al., 2005). En Santa Marta y San Andrés, entre el 1 y $30 \%$ del coral se blanqueó (ReefBase, 2006; Venera-Pontón, 2008; Wilkinson \& Souter, 2008). En el 2010 se desencadenó otro blanqueamiento atribuido a valores anómalos de temperatura (INAP, 2010). El Sistema Nacional de Monitoreo de Arrecifes de Coral (SIMAC), programa del INVEMAR, reportó en la estación de bahía Chengue (Santa
Marta) cerca de un $18 \%$ de corales blanqueados en ese año, por encima de los eventos de 1997 y 2005 (RodríguezRamírez, et al., 2008).

Debido a que en el blanqueamiento intervienen diferentes variables, es preciso establecer qué tan grave puede ser este en relación con el número de factores involucrados y sus distintos patrones espaciales (Glynn, 1993, Gil-Agudelo, et al., 2005, McClanahan, et al., 2007). Dicho de otro modo, las diferencias espaciales en la ocurrencia de este fenómeno pueden estar relacionadas con la interacción de variables y no únicamente con la TSM. En el Caribe colombiano los estudios se han limitado a mencionar posibles causas del blanqueamiento, sin profundizar en la combinación de las condiciones físicas asociadas (Zea \& Tobón, 1989; Solano, 1994; Rodríguez-Ramírez, et al., 2006; Wilkinson \& Souter, 2008; Navas-Camacho, et al., 2010). Ya que esta es una región donde la oceanografía marca diferencias que permiten contrastar la combinación de factores que inducen al blanqueamiento, en el presente estudio se utilizaron series históricas para evaluar algunas variables ambientales y relacionarlas con los blanqueamientos registrados a fin de entender sus causas.

\section{Metodología}

Se seleccionaron tres áreas de arrecifes en la plataforma continental y una en la zona oceánica del Caribe colombiano (Figura 1). Existen variaciones en la oceanografía de las localidades que dependen de la climatología de la región (Andrade \& Barton, 2005, Bernal, et al., 2006, Bernal, et al., 2010). Santa Marta está directamente influenciada por

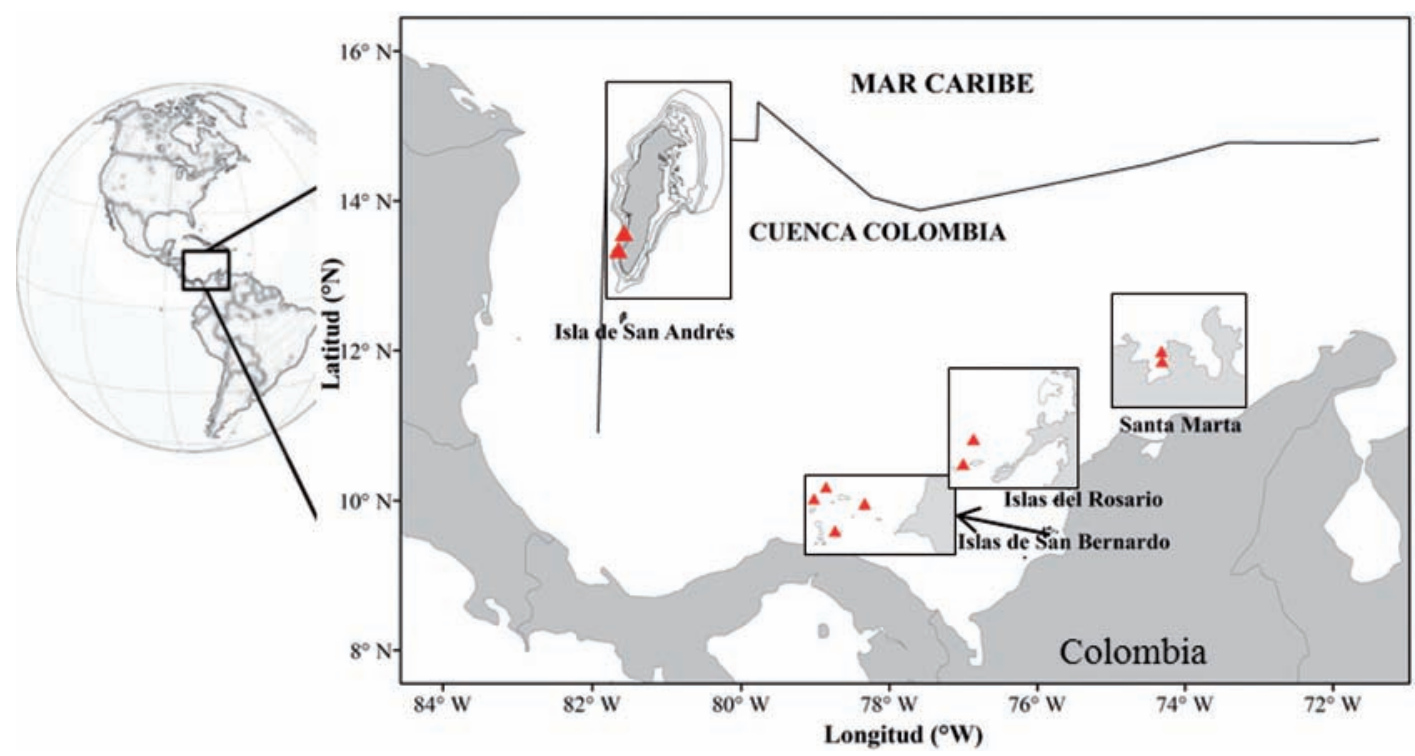

Figura 1. Área de estudio. Los triángulos señalan las estaciones del programa SIMAC de las cuales se obtuvo información de blanqueamiento coralino entre 1997 y 2010. 
los vientos del chorro de bajo nivel del Caribe y está sujeta a surgencias permanentes que varían en intensidad con el ciclo anual. Por su parte, la oceanografía de las islas del Rosario y de San Bernardo está dominada por el giro PanamáColombia. Allí se presentan aportes significativos de los ríos andinos, los cuales generan una estratificación del agua en la principal época lluviosa, lo cual aumenta el calentamiento superficial. El área de la isla de San Andrés, situada en el centro de la cuenca, está regida por la corriente del Caribe, sujeta a vientos más fuertes y expuesta al paso de huracanes (Andrade-Amaya, 2012).

Las series de tiempo de la temperatura superficial del mar (TSM), así como la información sobre la clorofila-a, el coeficiente de atenuación difusa $\left(\mathrm{kd}_{490}\right.$, medición indirecta de la turbidez), la velocidad del viento y la nubosidad, se obtuvieron de datos satelitales (Tabla 1). Asimismo, se adquirieron los datos del caudal de los ríos que desembocan cerca de los arrecifes bajo estudio en las estaciones del IDEAM más cercanas a la desembocadura de los ríos Piedras, Manzanares, Don Diego, Cañas y Gaira para el sector de Santa Marta; del Magdalena a través del canal del Dique para las islas del Rosario, y del Sinú para las de San Bernardo. Debido a que no hay series históricas de salinidad en la región, se usó información climatológica del World Ocean Atlas (WOA, 2009) y se interpretó mediante los datos de turbidez y caudal de los ríos. Estas variables se graficaron en mapas de promedio histórico, se les determinó el ciclo anual y se contrastaron con una prueba $\mathrm{T}$ para determinar diferencias significativas.

En cuanto al blanqueamiento, y puesto que no existen series históricas completas y la información se encuentra fragmentada, se usó una combinación de fuentes: 1) datos anuales del SIMAC sobre el porcentaje de coral vivo blanqueado desde 1998; 2) datos de ReefBase (sistema de información de arrecifes coralinos), y 3 ) información publicada sobre eventos de blanqueamiento en la región. Teniendo en cuenta que algunos registros de blanqueamiento (por ejemplo los de Reefbase), se clasifican según su intensidad alta, media y baja, las demás fuentes también se llevaron a estos intervalos mediante los valores medios, mínimos, máximos y las desviaciones estándar para el caso de los porcentajes. De esta manera se obtuvo una serie única de registros de blanqueamiento para cada localidad, la cual contiene los eventos reportados en las tres fuentes entre los años de 1997 y 2010, así como su intensidad. Es posible que hayan ocurrido eventos no registrados y que, por lo tanto, no aparezcan en la serie; sin embargo, se considera que esta sería la serie más completa de registros de blanqueamiento para la región.

Se hizo una aproximación de la relación entre las variables ambientales y el blanqueamiento a partir de los coeficientes factoriales obtenidos del análisis de componentes principales (ACP). Los coeficientes se graficaron como series de tiempo y se contrastaron con las fechas de blanqueamiento. El tiempo que los blanqueamientos de intensidad media y alta tardaron en evidenciarse, se evaluó con la revisión semanal detallada de las series de tiempo desde cuatro meses antes del primer reporte encontrado; el valor de las variables se clasificó como alto o extremo con base en las desviaciones estándar +1 y +2 , respectivamente; las gráficas no se muestran en este artículo, pero pueden encontrarse en Romero-Rodríguez (2013).

\section{Resultados}

\section{Caracterización espacio-temporal de las variables}

El gradiente espacial de los valores medios entre 1997 y 2010 de la TSM, la clorofila a (CLA), el $\mathrm{kd}_{490}$ y la salinidad se aprecia en la Figura 2. Hacia el noreste de la costa del Caribe colombiano, se identificaron las menores TSM; al sureste, en las islas del Rosario y San Bernardo, se identificaron TSM altas, y en el centro de la cuenca Colombia las aguas se caracterizaron por ser moderadamente cálidas. El borde costero se encontró mesotrófico (CLA $0,2-1,0 \mathrm{mg} / \mathrm{m}^{3}$ ) y con mayor $\mathrm{kd}_{490}$, mientras que el centro de la cuenca Colombia se evidenció como oligotrófico (CLA $<0,2 \mathrm{mg} / \mathrm{m}^{3}$ ) y con menor $\mathrm{kd}_{490}$. Por su parte, la salinidad media fue menor en las localidades costeras en comparación con la zona oceánica y fue mayor en la parte noreste que en la parte sureste de la cuenca Colombia.

Tabla 1. Fuente y resolución de las variables ambientales utilizadas en el presente estudio, promedios semanales (septiembre de 1997 a diciembre de 2010)

\begin{tabular}{|c|c|c|c|}
\hline Variable & Fuente & Resolución especial & Página Web \\
\hline Temperatura superficial del $\operatorname{mar}\left({ }^{\circ} \mathrm{C}\right)$ & NOAAAVHRR & $4 \mathrm{~km}$ & http://www.nodc.noaa.gov/SatelliteData/pathfinder $4 \mathrm{~km} /$ \\
\hline Clorofila $\left(\mathrm{mg} / \mathrm{m}^{3}\right)$ y turbidez $\left(\mathrm{m}^{-1}\right)$ & SeaWiFS & $9 \mathrm{~km}$ & http://oceancolor.gsfc.nasa.gov/ \\
\hline Velocidad del viento $(\mathrm{m} / \mathrm{s})$ & ССMP & $0,25^{\circ}$ & http://podaac.jpl.nasa.gov/dataset/ \\
\hline Nubosidad (\%) & NCEP/NCAR & $2^{\circ}$ & http://www.esrl.noaa.gov/psd/data/gridded/data.nmc.marine.html \\
\hline Salinidad superficial del mar & WOA2009 & $0,25^{\circ}$ & http://www.nodc.noaa.gov/OC5/WOA09/pr_woa09.html \\
\hline
\end{tabular}

NOAA: National Oceanic and Atmospheric Administration, AVHRR: Advanced Very High Resolution Radiometer, SeaWiFS: Sea-Viewing Wide Field-ofView Sensor, CCMP: Cross-Calibrated Multi-Platform, NCEP/NCAR: National Centers for Environmental Prediction/ National Center for Atmospheric Research, WOA= World Ocean Atlas 

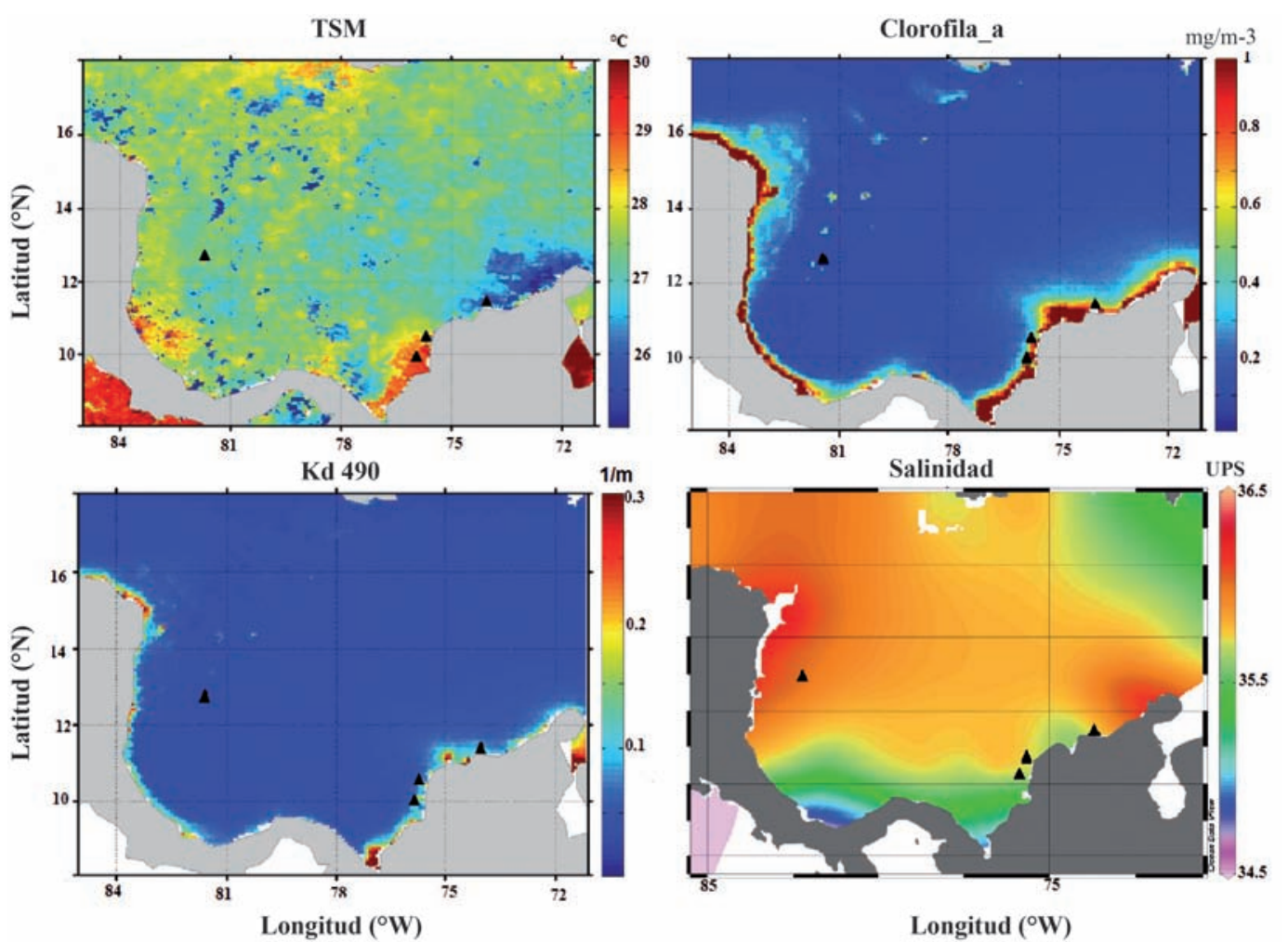

Figura 2. Valores medios de $\operatorname{TSM}\left({ }^{\circ} \mathrm{C}\right)$, clorofila $(\mathrm{mg} / \mathrm{m} 3)$ y $\mathrm{kd}_{490}\left(\mathrm{~m}^{-1}\right)$ entre 1997 y 2010 , y salinidad (climatología del WOA). Los triángulos indican las localidades de estudio.

El comportamiento de las variables durante el año se muestra en la Figura 3. De diciembre a abril, la TSM, la clorofila a, el $\mathrm{kd}_{490}$, el caudal de los ríos y el porcentaje de nubosidad presentaron valores bajos, mientras que los vientos fueron altos. Entre mayo y noviembre, con interrupciones en julio y agosto, las variables tuvieron valores altos, a excepción de los vientos, que fueron débiles. Los valores medios del ciclo de la TSM en las islas del Rosario y San Bernardo, no presentaron una diferencia significativa (valor $\mathrm{p}>0,05$ ). Los menores valores se identificaron en Santa Marta y en la isla de San Andrés, y tampoco presentaron diferencias significativas entre sus medias. Respecto a la clorofila a, los valores más altos se identifican en los sitios costeros (Santa Marta, islas del Rosario y San Bernardo), sin diferencias significativas entre ellos. El caso contrario se presentó en la isla de San Andrés, con diferencias significativas frente a los sitios costeros (valor $\mathrm{p}=0,00$ ). El caudal no presentó diferencias significativas entre las medias de San Bernardo e islas del Rosario (valor $\mathrm{p}=0,9$ ), sin embargo, el de Santa Marta sí tuvo diferencias con respecto al de islas del Rosario y San Bernardo. Los vientos más intensos se registraron en la isla de San Andrés y en Santa Marta, sin diferencias significativas, así como tampoco las hubo entre San Bernardo e islas del Rosario (valor $\mathrm{p}=0,12$ ).

\section{Identificación de los blanqueamientos coralinos en el área de estudio}

Los blanqueamientos reportados entre 1997 y 2010 se muestran en la Figura 4. En Santa Marta e islas del Rosario se presentaron tres blanqueamientos de intensidad alta y tres de intensidad media entre los meses de agosto a diciembre. En San Bernardo se identificaron dos blanqueamientos de intensidad media y uno de intensidad alta en meses diferentes (marzo y junio); no obstante, la serie de blanqueamientos de San Bernardo fue más corta, probablemente porque la cantidad de estudios realizados allí ha sido menor. En la isla de San Andrés se encontraron tres blanqueamientos de intensidad media y uno de intensidad alta entre mayo y octubre. Los blanqueamientos se dieron en tres escalas espaciales: de cuenca, en 1998 y 2005, ocurridos tanto en las áreas costeras como en la oceánica; costera, en 1999, 2003 y 2010, ocurridos en al menos dos de las tres áreas de la plataforma continental, y local, en 2002, 2004, 2006, 2008 y 2009, ocurridos solamente en una de las áreas.

\section{Características ambientales en los blanqueamientos coralinos}

En los tres sectores costeros, la componente uno del ACP estuvo dominada por la correlación positiva con los vientos y la negativa con la temperatura, el caudal, la clorofila a y el $\mathrm{kd}_{490}$ (Tabla 2). En Santa Marta, los picos negativos de la serie de 

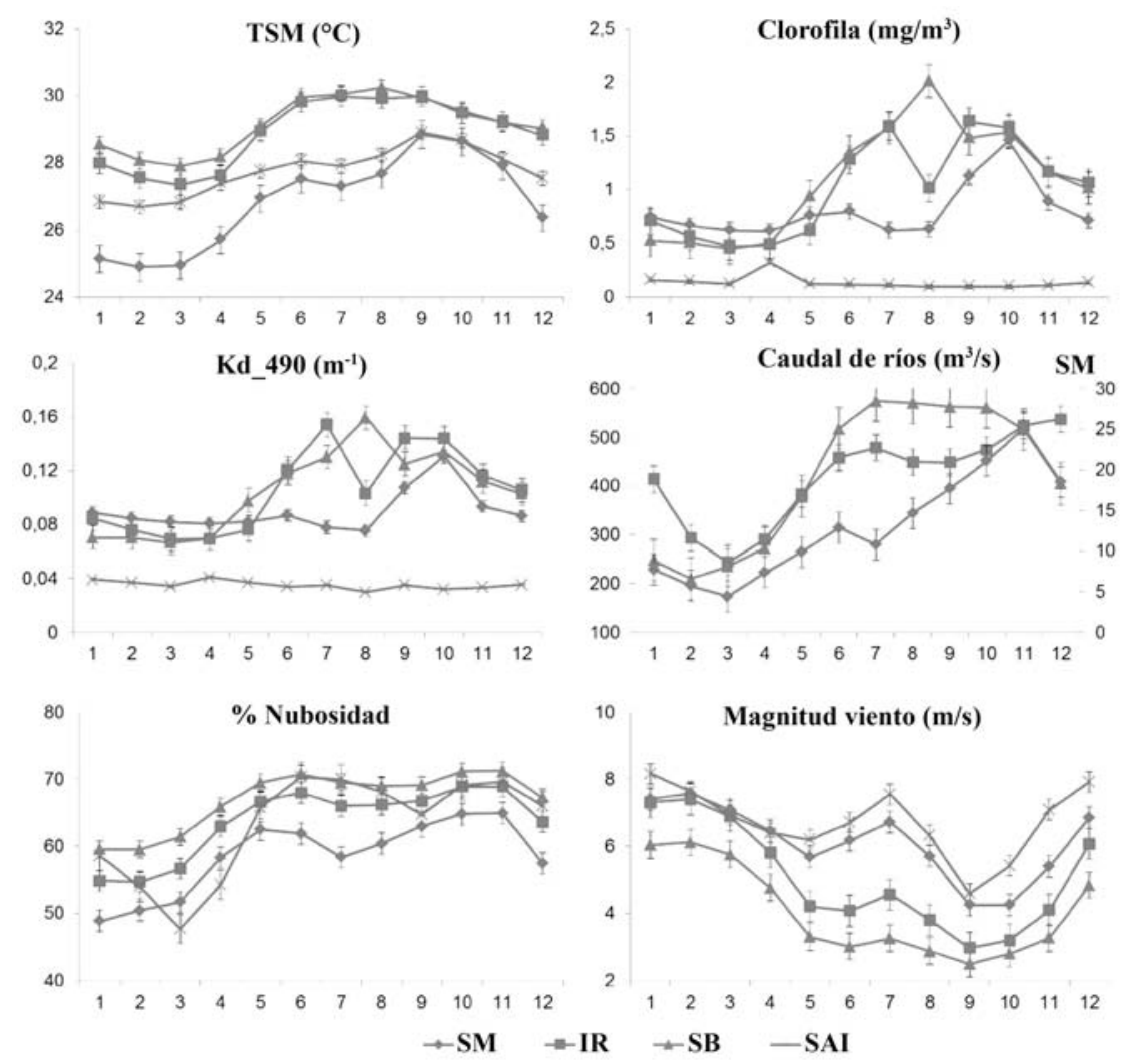

Figura 3. Promedios mensuales de la serie temporal completa entre 1997 y 2010. TSM (PathfinderVersion 5.2); clorofila y kd ${ }_{490}$ (sensor SeaWiFS); caudal de ríos (IDEAM); nubosidad (NCEP/NCAR), y vientos (CCMP) SM: Santa Marta, IR: Islas del Rosario, SB: San Bernardo, SAI: Isla de San Andrés
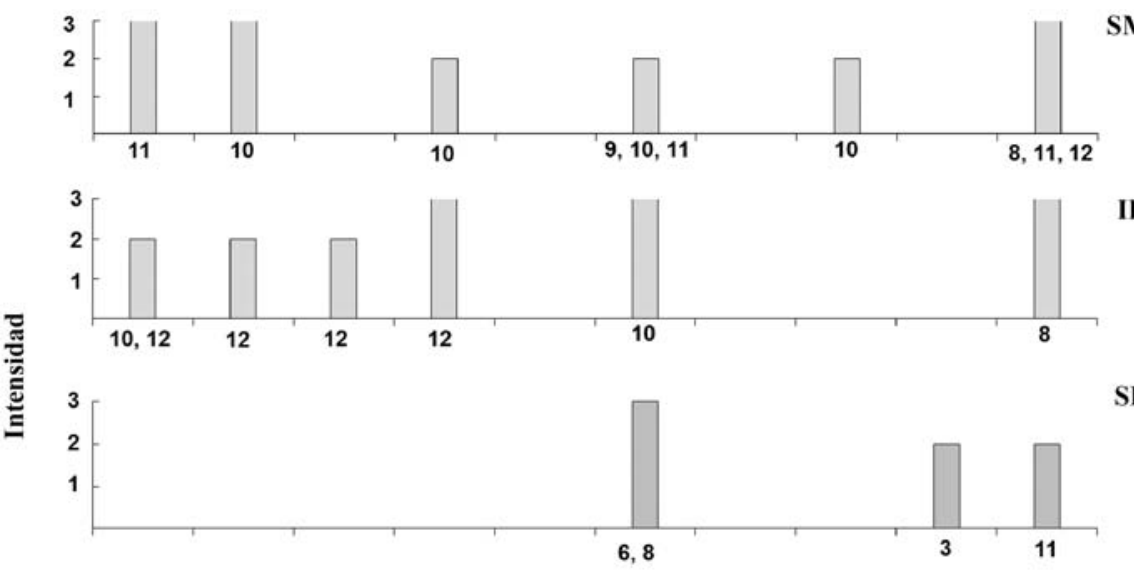

IR

SB

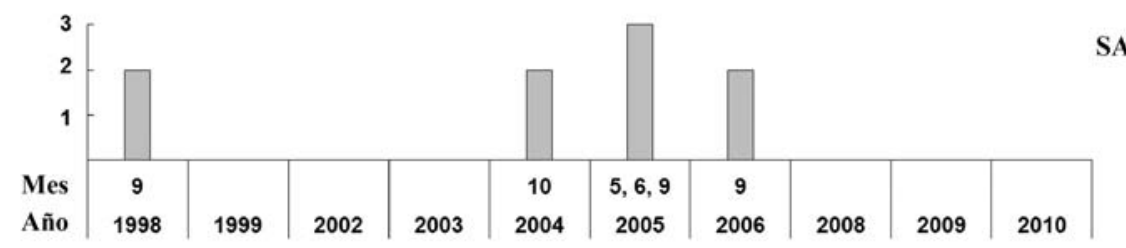

Figura 4. Intensidad del blanqueamiento entre 1997 y 2010 a partir de diferentes fuentes de información. Los meses en que se registró blanqueamiento se indican con números bajo las barras. SM: Santa Marta, IR: Islas del Rosario, SB: San Bernardo, SAI: Isla de San Andrés 
Tabla 2. Coeficientes factoriales de las dos primeras componentes, las cuales explican más del $70 \%$ de la varianza en cada sector

\begin{tabular}{lcccccccc}
\hline Variable & \multicolumn{2}{c}{ Santa Marta } & \multicolumn{2}{c}{ Islas del Rosario } & \multicolumn{2}{c}{ San Bernardo } & \multicolumn{2}{c}{ Isla de San Andrés } \\
\cline { 2 - 8 } & C 1 & C 2 & C 1 & C 2 & C 1 & C 2 & C 1 & C 2 \\
\hline Caudal $\left(\mathrm{m}^{3} / \mathrm{s}\right)$ & $-0,68$ & 0,25 & $-0,61$ & $-0,03$ & $-0,81$ & 0,28 & \\
Nubosidad $(\%)$ & $-0,68$ & 0,28 & $-0,57$ & $-0,45$ & $-0,58$ & 0,3 & $-0,17$ & 0,39 \\
TSM $\left({ }^{\circ} \mathrm{C}\right)$ & $-0,79$ & 0,37 & $-0,67$ & $-0,45$ & $-0,65$ & 0,44 & 0,6 & 0,72 \\
\hline Vientos $(\mathrm{m} / \mathrm{s})$ & 0,80 & $-0,27$ & 0,72 & 0,47 & 0,84 & $-0,27$ & 0,61 & $-0,49$ \\
Clorofila $\left(\mathrm{mg} / \mathrm{m}^{3}\right)$ & $-0,53$ & $-0,84$ & $-0,82$ & 0,55 & $-0,76$ & $-0,6$ & 0,77 & 0,52 \\
$\mathrm{Kd}_{490}\left(\mathrm{~m}^{-1}\right)$ & $-0,49$ & $-0,87$ & $-0,76$ & 0,62 & $-0,74$ & $-0,62$ & 0,73 & 0,56 \\
\hline
\end{tabular}

C1: Componente 1, C2: Componente 2, del Análisis de Componentes Principales
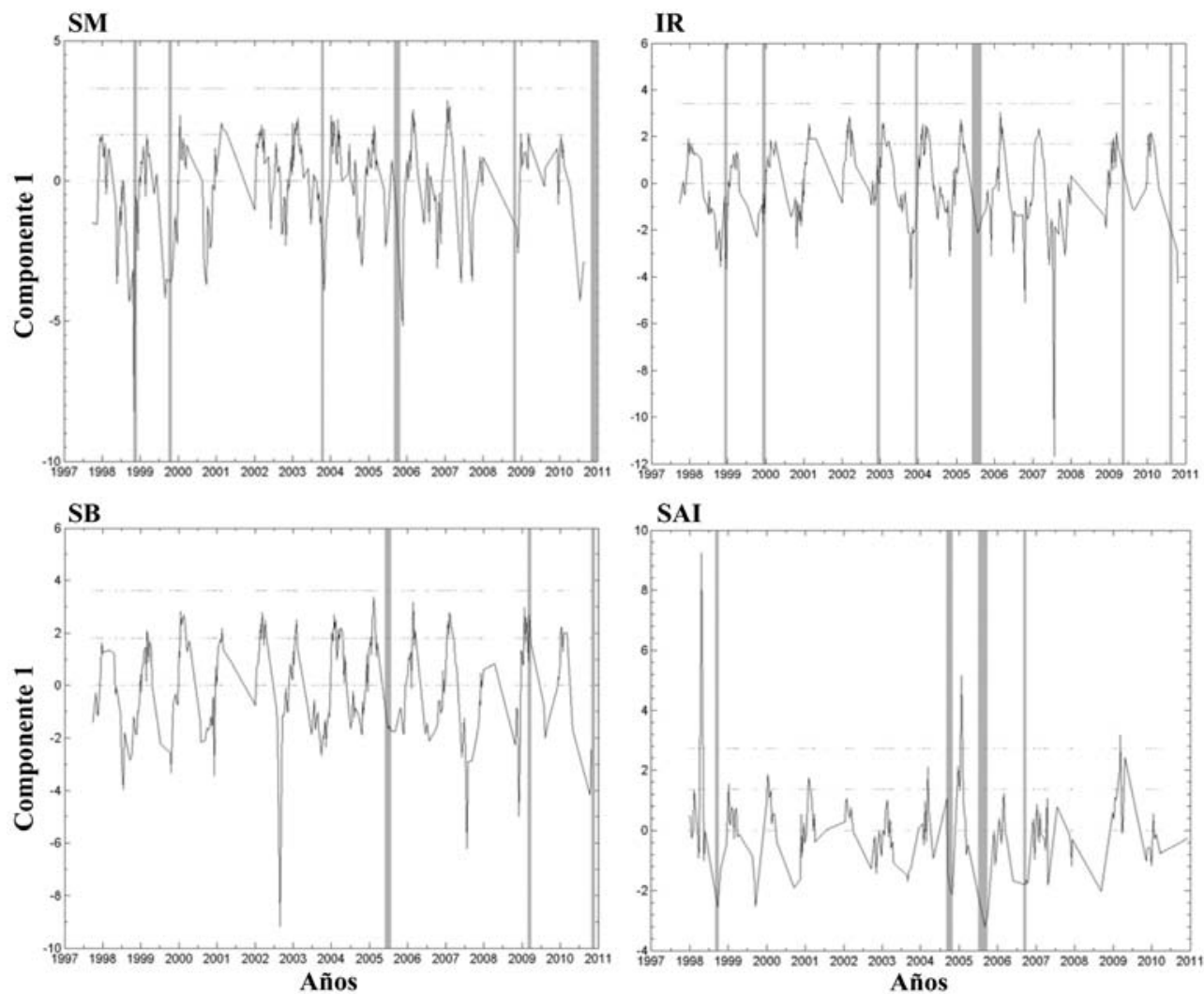

Figura 5. Serie de tiempo de la componente uno del ACP en cada área de estudio. Las barras grises indican los meses en que hubo blanqueamiento de intensidad media o alta. SM: Santa Marta, IR: Islas del Rosario, SB: San Bernardo, SAI: Isla de San Andrés

tiempo de los coeficientes factoriales de la componente uno, coincidieron con los blanqueamientos de 1998, 1999, 2003, 2005 y 2008 (Figura 5), es decir que los blanqueamientos coincidieron con aumentos combinados de la TSM, el caudal, la clorofila a y el $\mathrm{kd}_{490}$. El análisis de la serie de tiempo desde cuatro meses antes indicó que en Santa Marta la TSM, la clorofila a, el $\mathrm{kd}_{490} \mathrm{y}$ el caudal fueron extremos desde por lo menos un mes antes de los blanqueamientos de intensidad alta. En las islas del Rosario, los blanqueamientos de 1998,
1999, 2003, 2005 y 2010 coincidieron con picos negativos de la componente principal (alta TSM, CLA, $\mathrm{kd}_{490} \mathrm{y}$ caudal). En este sector, la TSM, la clorofila y la turbidez fueron extremas desde por lo menos dos meses antes de cada uno de los blanqueamientos. En San Bernardo, los blanqueamientos de 2005 y 2010 estuvieron asociados con estos picos. En el blanqueamiento del 2005 la TSM, la clorofila y la turbidez fueron altas un mes antes, mientras que en el blanqueamiento del 2010 las variables fueron extremas desde por lo menos 
tres meses antes. Por su parte, en la isla de San Andrés las dos primeras componentes principales representaron el 67,4\% y se relacionaron con la turbidez y la clorofila $(\mathrm{C} 1)$ y la TSM (C2); así, los blanqueamientos de 1998, 2003, 2005 y 2006 coincidieron con una disminución de la turbidez y la clorofila y un aumento de la temperatura (Figura 5). Allí la temperatura fue extrema en todos los blanqueamientos desde por lo menos un mes antes y sólo se identificó un valor extremo de turbidez dos semanas antes del blanqueamiento de 2004.

\section{Discusión}

La oceanografía en los cuatro sectores presenta variaciones espacio-temporales relacionadas con la estacionalidad propia de la cuenca Colombia (Mesa, et al., 1997; Bernal, et al., 2006; Ruiz-Ochoa, et al., 2012; Bayraktarov, et al., 2014). A pesar de que los datos satelitales presentan limitaciones temporales y espaciales en la captura de información, y que algunas de las variables deben ser calibradas si se quiere trabajar con valores absolutos, la información obtenida fue suficiente para identificar las diferencias entre las localidades y las condiciones relativas de las variables ambientales durante los eventos de blanqueamiento.

Por su parte, los datos de blanqueamiento deben analizarse cuidadosamente debido a la combinación de fuentes y a que la monitorización del SIMAC es anual y no siempre coindice con el momento en que ocurre el blanqueamiento (Vega-Sequeda, et al., 2011). Los valores del SIMAC son indicativos de que hubo blanqueamiento en algún momento antes o durante la monitorización, pero estos datos no se pueden correlacionar directamente con los valores contemporáneos de las variables ambientales. Los blanqueamientos encontrados no son necesariamente todos los que han ocurrido en la región, por lo tanto, los intentos de relacionar los eventos de blanqueamiento con causas particulares siguen viéndose obstaculizados por la falta de métodos estandarizados para evaluar el blanqueamiento y de bases de datos continuos y de largo plazo. Por ello se propone incrementar las épocas de monitorización de los blanqueamientos por parte de las instituciones y de la propia comunidad de cada sector, que puede recibir entrenamiento con base en los manuales ofrecidos por programas como el de Reef Check (http://reefcheckdr.org/).

En la zona costera del Caribe colombiano la variabilidad anormal de la TSM y de las descargas continentales (agrupa-

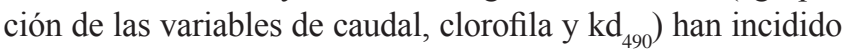
en el blanqueamiento de los corales y en la escala espacial con que se produce. Las diferencias en la intensidad del blanqueamiento de los corales pueden estar asociadas al tiempo de exposición a valores anómalos. Cuando solo la temperatura o el caudal fueron altos, los blanqueamientos tuvieron una intensidad media, pero cuando ambas variables se mantuvieron altas durante aproximadamente dos meses, se desencadenaron blanqueamientos de intensidad alta. También se identificaron situaciones en las que ambas variables fueron altas, pero su permanencia no excedió los dos meses y los blanqueamientos asociados fueron de intensidad media. Por otra parte, en el sitio insular la única variable asociada fue la temperatura.

En las islas del Rosario y San Bernardo la mayor turbidez (medida indirectamente por medio del $\mathrm{kd}_{490}$ ), se presenta durante los meses de septiembre a noviembre, lo cual expone a los arrecifes a altos niveles de sedimentos y nutrientes que podrían afectar a las especies y producir cambios en la respuesta de los organismos al blanqueamiento (Williams \& Bunkley, 1990; Coles \& Brown, 2003; West \& Salm, 2003; McClanahan, et al., 2007; Devlin, et al., 2012). En estos sectores hay mayor riesgo de que ocurran blanqueamientos por estrés térmico, ya que la temperatura promedio está cercana a la temperatura umbral del blanqueamiento en los corales $\left(29^{\circ} \mathrm{C}\right)$ (Kleypas, et al., 2001), en tanto que la región de menor probabilidad es Santa Marta, ya que la surgencia durante la época seca aumenta la salinidad y disminuye la temperatura, propiciando condiciones favorables para la salud coralina (Gil-Agudelo, 2005; Bayraktarov, et al., 2013).

Los eventos macroclimáticos y los huracanes generan efectos combinados en las variables ambientales, lo que deja a los corales aún más deteriorados y expuestos (Buddemeier, $\boldsymbol{e t}$ al., 2004; Maina, et al., 2008). En el Caribe colombiano se han reportado blanqueamientos significativos de los arrecifes coralinos localizados en el área costera durante los eventos de La Niña de 1998-2000 y 2007-2008, fase en la cual se produjo un incremento de la precipitación y los caudales en Colombia (IDEAM, 1998, 2008). No hubo blanqueamientos durante El Niño a pesar de su asociación con aumentos de la temperatura, lo que puede deberse a que la costa Caribe colombiana tiene un rezago de hasta seis meses en la respuesta de la TSM con respecto al Pacífico (Bernal, et al. 2006), o a que esta no se combinó con altos caudales. De otro lado, el blanqueamiento coralino puede dejar al arrecife más expuesto a los efectos negativos de un huracán, como sucedió en el caso del huracán Lenny en 1999, el cual disminuyó la cobertura coralina de bahía Chengue en un $32 \%$ (afectando especialmente a Acropora palmata), como consecuencia de los daños físicos por fragmentación, rayones y volcamiento (Garzón-Ferreira \& Díaz, 2003).

\section{Conclusiones}

En el Caribe colombiano los corales están expuestos a la presión que ejercen los eventos naturales extremos, los que combinados con los de naturaleza antropogénica, disminuyen la calidad del agua, dejando a los corales más expuestos a blanqueamientos. A pesar de las limitaciones de la información satelital, se estableció que en el sector oceánico la temperatura 
juega el papel principal en el blanqueamiento, mientras que en los sitios costeros, las descargas continentales influyen de manera significativa en la intensidad.

Las condiciones ambientales más propicias para eventuales blanqueamientos coralinos se encontraron en las islas del Rosario y San Bernardo; allí se presentaron altas temperaturas, abundante entrada de agua dulce y turbidez, lo cual se puede asociar con el incremento del porcentaje de blanqueamiento desde 2005 hasta la fecha.

Los blanqueamientos a escala de la cuenca se han relacionado especialmente con el aumento en la temperatura superficial del mar, mientras que en los blanqueamientos a escala continental y local, la temperatura y el caudal han sido los principales factores, lo que involucra, igualmente, el tiempo de exposición de los corales a valores anómalos.

\section{Agradecimientos}

A la Universidad Nacional de Colombia, por la financiación del proyecto "Alianza para el estudio del impacto de los eventos extremos sobre los ecosistemas marino costeros insulares"; a Colciencias y a la Universidad Nacional de Colombia, por la financiación del proyecto "Eventos oceánicos extremos en ecosistemas costeros insulares del Pacífico y Caribe colombianos" (proyecto 1118-569-34826), al programa SIMAC del INVEMAR por los datos aportados y al Centro de Estudios en Ciencias del Mar - CECIMAR, Universidad Nacional de Colombia, Sede Caribe, por la contribución en las bases de datos. Agradecemos, igualmente, al Dr. Emilio Beier del Centro de Investigación Científica y Educación Superior de Ensenada (Cicese), La Paz, México, por su asesoría y comentarios.

\section{Conflicto de interés}

Los autores declaran que no tienen ningún conflicto de interés.

\section{Referencias}

Andrade-Amaya, C. A. 2012. Oceanografía del archipiélago de San Andrés, Providencia y Santa Catalina. En: Atlas de la Reserva de Biósfera Seaflower. Archipiélago de San Andrés, Providencia y Santa Catalina. Gómez-López, D. I., C. Segura-Quintero, P. C. Sierra-Correa y J. Garay-Tinoco (eds.). Instituto de Investigaciones Marinas y Costeras “José Benito Vives De Andréis" -INVEMAR- y Corporación para el Desarrollo Sostenible del Archipiélago de San Andrés, Providencia y Santa Catalina -CORALINA-. Serie de Publicaciones Especiales de INVEMAR \# 28. Santa Marta, Colombia. 180 p.

Andrade C. \& Barton E. 2005. The Guajira upwelling system. Continental Shelf Research 25: 1003-1022.

Bayraktarov, E., Pizarro, V., Eidens, C., Wilke, T., Wild, C. 2013. Bleaching susceptibility and recovery of Colombian Caribbean corals in response to water current exposure and seasonal upwelling. PloS one 8 (11): e80536.
Bayraktarov, E., \& Wild, C. 2014. Spatiotemporal variability of sedimentary organic matter supply and recycling processes in coral reefs of Tayrona National Natural Park, Colombian Caribbean. Biogeosciences 11 (11): 2977-2990.

Bernal, G., Poveda, G., Roldán, P., Andrade, C. 2006. Patrones de variabilidad de las temperaturas superficiales del mar en la costa Caribe colombiana. Rev. Acad. Colomb. Cienc. 30 (115): 195-208.

Bernal, G., Ruiz-Ochoa, M. \& Beier, E. 2010. Variabilidad estacional e interanual océano-atmósfera en la cuenca Colombia. En: La investigación en Ciencias del Mar de la Universidad Nacional de Colombia. 30 años de la Biología Marina. Campos, N.H., Acero, A., Mancera, J.E. (eds.). Universidad Nacional de Colombia, Bogotá. Cuadernos del Caribe 14: 49-72.

Brown, B. E. 1997. Coral bleaching: Causes and consequences. Coral Reefs 16: S129-S138.

Buddemeier, R.W., Kleypas J.A. \& Aronson, R.B. 2004. Coral reefs and global climate change. Potential contributions of climate change to stress on coral reef. Pew Center on Global Climate Change. Virginia, USA. 44 p.

Coles, S. L., \& Brown, B. E. 2003. Coral bleaching-capacity for acclimatization and adaptation. Advances in marine biology 46: 183-223.

Devlin, M., Wenger, A., Da Silva, E., Álvarez Romero, J. G., Waterhouse, J., McKenzie, L. 2012. Extreme weather conditions in the Great Barrier Reef: Drivers of change. Proceedings of the 12th International Coral Reef Symposium, Cairns, QLD, Australia. 5 pag.

Eakin, C. M., Morgan, J. A., Heron, S. F., Smith, T. B., Liu, G., Álvarez-Filip, L., Quinn, N. 2010. Caribbean corals in crisis: Record thermal stress, bleaching, and mortality in 2005. PloS one 5 (11): e13969.

Douglas, A. E. 2003. Coral bleaching -how and why? Marine Pollution Bulletin 46 (4): 385-392.

Gardner, T. A., Cote, I. M., Gill, J. A., Grant, A., Watkinson, A. R. 2005. Hurricanes and Caribbean coral reefs: Impacts, recovery patterns, and role in long-term decline. Ecology 86: 174-184.

Garzón-Ferreira, J. \& Díaz. J. M. 2003. The Caribbean coral reefs of Colombia. En J. Cortés (ed.). Latin American coral reefs. Elsevier Science B.V., Amsterdam, the Netherlands, pp. 275-301.

Gil-Agudelo, D.L., Garzón-Ferreira, J., Rodríguez-Ramírez, A., Reyes-Nivia, M. C., Navas-Camacho, R., Venera-Pontón, D. E., Díaz-Pulido, G., Sánchez, J. A. 2005. Estado de los arrecifes coralinos en Colombia. En: Informe del Estado de los Ambientes Marinos y Costeros de Colombia: 2005. Serie de publicaciones periódicas del INVEMAR, No. 8, Santa Marta. 360 pp.

Glynn, P. W. 1993. Coral reef bleaching: Ecological perspectives. Coral Reefs 12 (1): 1-17. 
Hoegh-Guldberg, O. 1999. Climate change, coral bleaching and the future of the world's coral reefs. Marine and fresh water research 50 (8): 839-866.

Kleypas, J. A., Buddemeier, R. W. \& Guttuso. J. 2001.The future of coral reefs in an age of global change. International Journal of Earth Sciences 90: 426-437.

Maina, J., Venus, V., McClanahan, T. R., Ateweberhan, M. 2008. Modelling susceptibility of coral reefs to environmental stress using remote sensing data and GIS models. Ecological modelling 212 (3): 180-199.

McClanahan, T., Ateweberhan, M., Muhando, C., Maina, J., Mohammed, J. 2007. Effects of climate and seawater temperature variation on coral bleaching and mortality. Monografías Ecológicas 77 (4): 503-525.

Mumby, P. J., Chisholm, J. R. M., Edwards, A. J., Andrefouet, S., Jaubert, J. 2001. Cloudy weather may have saved Society Island reef corals during the 1998 ENSO event. Marine Ecology-Progress Series 222: 209-216.

Navas-Camacho, R., Gil-Agudelo, D.L., Rodríguez-Ramírez, A., Reyes-Nivia, M.C., Garzón-Ferreira, J. 2010. Coral diseases and bleaching on Colombian Caribbean coral reefs. Rev. Biol. Trop. 58 (Suppl. 1): 95-106.

Ruiz-Ochoa, M., Beier, E., Bernal, G., E.D. Barton. 2012. Sea surface temperature variability in the Colombian Basin, Caribbean Sea. Deep Sea Research I 64: 43-53.

Solano, P. O. D. 1994. Corales, formaciones arrecifales y blanqueamiento de 1987 en Bahia Portete, Guajira, Colombia. Anales del Instituto de Investigaciones Marinas de Punta de Betín (Santa Marta) 23: 149-163.

True, J. D. 2012. Salinity as a structuring force for near shore coral communities. Proceedings of the 12th International Coral Reef Symposium, Cairns, Australia.

Van Woesik, R., De Vantier, L. M. \& Glazebrook. 1995. Effects of Cyclone 'Joy' on nearshore coral communities of the Great Barrier Reef. Series de Ecología Marina 128: 261-270.

West, J.M. \& Salm, R.V. 2003. Resistance and resilience to coral bleaching: Implications for coral reef conservation and management. Conservation Biology 17 (4): 956-967.

Wiedenmann, J., D’Angelo, C., Smith, E.G., Hunt, A.N., Legiret, F. E., Postle, A.D., Achterberg, E.P. 2013. Nutrient enrichment can increase the susceptibility of reef corals to bleaching. Nature Climate Change 3: 160-164.

Wilkinson, C. \& Souter, D. 2008. Status of Caribbean coral reefs after bleaching and hurricanes in 2005. Global Coral Reef Monitoring Network and Reef and Rainforest Research Centre, Townsville, $152 \mathrm{pp}$.

Williams E. H \& Bunkley - Williams L. 1990. The world-wide coral reef bleaching cycle and related sources of coral mortality. Atoll Res Bull 335: 1-71.

Zea, S. \& Tobón, D. 1989. Bleaching of reef organisms in the Santa Marta region, Colombia: 1987 Caribbean-wide event. Trianea (Act. Cient. Técn., INDERENA) 3: 37-75. 